\title{
La congélation de l'embryon humain
}

La congélation de l'embryon humain offre désormais des chances supplémentaires de succès dans la lutte contre l'infertilité. Ces techniques relancent le débat éthique qui a accompagné chacune des innovations de ce secteur.

\section{Jean-Paul Renard Chargé de recherches à l'Inra.}

Note de la Rédaction

Le texte de Fean-Paul Renard décrit la technique de congélation des embryons utilisée depuis quelques années pour la reproduction des animaux domestiques et appliquée récemment dans l'espèce humaine. Le Comité de Rédaction est conscient des problèmes ethiques importants que soulève l'application de cette technique chez l'homme. Ce texte doit être considéré comme une introduction à la réflexion sur ces problèmes.

\section{ADRESSE}

Jean-Paul Renard : Unité de génétique des mammiferes, Institut Pasteur, 25, rue du Docteur-Roux, 75724 Paris cedex 15.

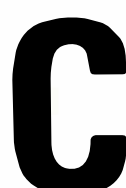
e fut d'abord une petite fille qui naquit en Australie le i I avril 1984 , et on parla beaucoup d'elle dans le monde entier. D'autres bébés " plus discrets " suivirent et aujourd'hui ils sont environ une quinzaine d'enfants à vivre après avoir connu l'état congelé au tout début de leur vie embryonnaire.

Ce qui pour beaucoup apparaît avant tout comme un nouvel exploit d'équipes biomédicales audacieuses, est en fait l'aboutissement logique des progrès réalisés ces dernières années dans le domaine de la conservation des embryons de mammiferes.En effet, en $\mathbf{1 9 7 2}$, il a été établi pour la première fois [I] que des embryons de souris congelés au stade 8 cellules, à des températures aussi basses que $-269^{\circ} \mathrm{C}$, pouvaient une fois décongelés se développer normalement et donner des jeunes viables après transfert dans l'utérus d'une femelle receveuse. Depuis, de nombreuses recherches ont été effectuées chez plusieurs espèces, notamment les bovins où la congélation de l'embryon commence à être utilisée pour préserver et diffuser les gènes des meilleures femelles issues des programmes de sélection. Dans le présent article, nous montrerons, après avoir présenté les données fondamentales de la cryobiologie, que c'est en transposant à l'homme les méthodes de conservation définies chez l'animal que " ces enfants du froid ", par ailleurs semblables aux autres ont pu voir le jour. Bien que les méthodes proposées aujourd'hui chez l'homme aient une ef ficacité encore faible, on constate que déjà la congélation de l'embryon devient une nouvelle technique médicale pour les équipes qui pratiquent la fécondation in vitro et le transfert embryonnaire (FIVETE). Il importe alors de s'interroger sur l'avenir de cette technique qui, en permettant une dissociation complète dans le temps entre la conception et la naissance, pose de nouveaux problèmes éthiques.

\section{La cryobiologie}

La réponse des cellules embryonnaires aux eff ets de la congélation et de la décongélation est semblable à celle de cellules aussi différentes que les érythrocytes ou les micro-organismes : leur survie dépend essentiellement des conditions du changement de phase de l'eau cellulaire et de l'action des cryoprotecteurs.

Dès qu'un premier cristal de glace apparaît dans le milieu de suspension des cellules, la concentration en solutés dissous augmente rapidement et la température de congélation diminue (loi de Raoult) : l'équilibre osmotique avec le milieu intérieur se rétablit par un mouve- 
ment de l'eau hors de la cellule. Les mouvements de l'eau dépendent de plusieurs paramètres, notamment de la taille de la cellule (rapport surface-volume qui définit la surface relative d'échanges avec le milieu extérieur), de sa conductivité hydraulique (perméabilité des membranes plasmiques) et de son énergie d'activation (c'est-à-dire de la variation de la conductivité hydraulique avec la température) [2]. Le milieu cellulaire ne va donc pas se congeler brutalement à partir d'une température donnée, mais progressivement sur un intervalle de température (plusieurs dizaines de degrés) pendant lequel la concentration en solutés augmente au fur et à mesure que la cellule se déshydrate (figure I).

Si la déshydratation est trop importante, la concentration élevée en électrolytes provoque des modifications biochimiques du milieu cellulaire, modifications connues sous le nom "d'effets de solution" [3]. Si la déshydratation est insuffisante, des cristaux de glace se forment à l'intérieur des cellules [4] : une fine trame de glace s'interpénètre dans une phase vitreuse (sans cristaux) avec, même à très basse température $\left(-40^{\circ} \mathrm{C}\right)$, une fraction non congelée soumise à des gradients élevés de concentration en solutés. Au cours du réchauffement, les cristaux de glace se transforment avant de fondre : les molécules d'eau passent de la surface des petits cristaux à celle des plus grands dont la taille s'accroît rapidement, endomma-

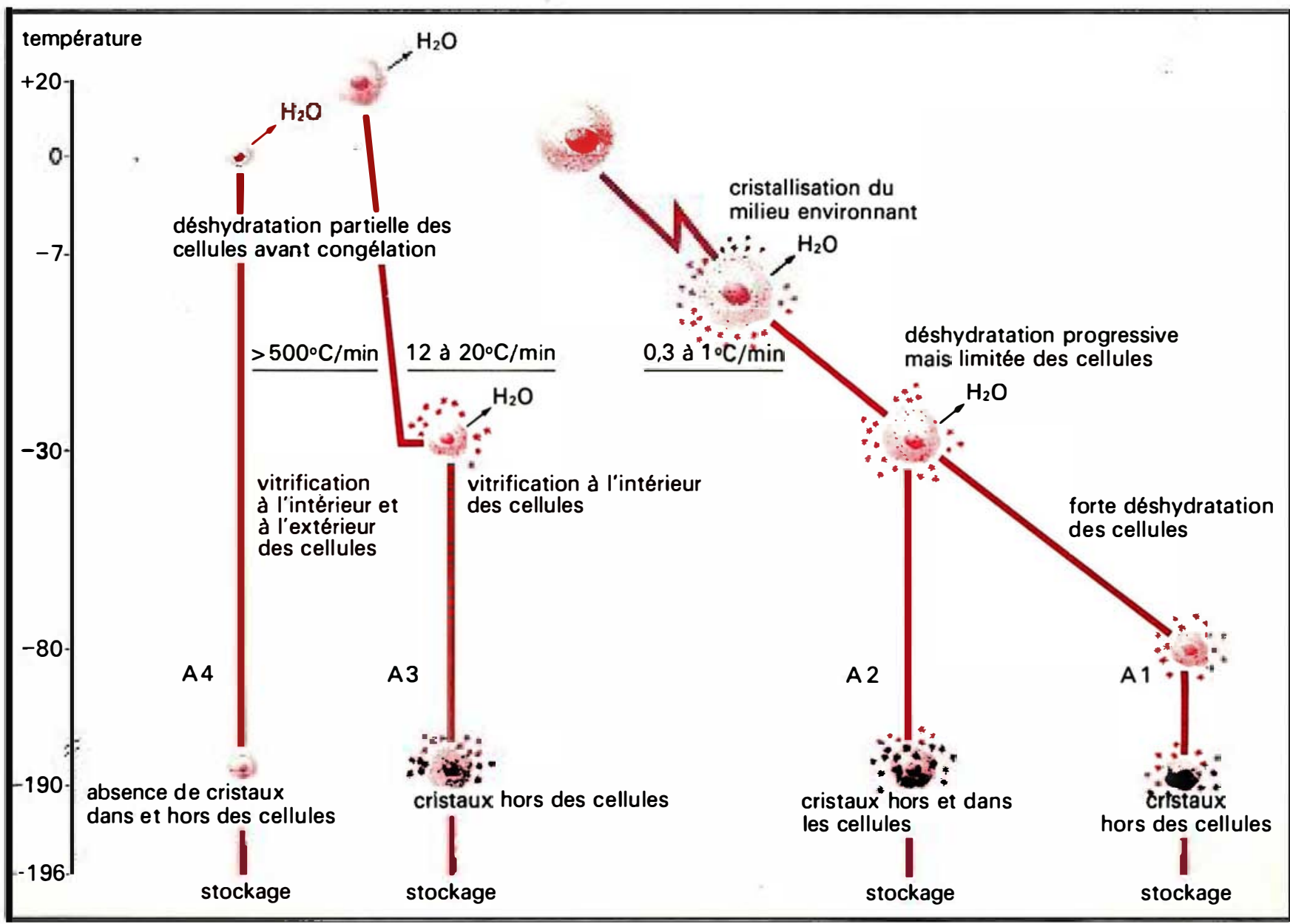

Figure r. Méthodes utilisées pour la congélation des embryons de mammiferes. Ces méthodes differrent les unes des autres par la vitesse de congélation et la nature des cryoprotecteurs employés. Une vitesse de congélation lente de $0,3^{\circ} \mathrm{C}$ à $I^{\circ} \mathrm{C} /$ min permet une déshydratation progressive des embryons au cours de l'abaissement de la température (méthodes AI et A2). Les effets de cette déshydratation s'ajoutent à ceux produits par le remplacement d'une fraction de l'eau cellulaire par un cryoprotecteur (ex : glycerol, DMSO) qui, en pénétrant dans les cellules, peut empêcher la formation de cristaux de glace (méthode AI) ou en limiter l'importance (méthode A2). En exposant les embryons avant refroidissement à une solution hyperosmotique contenant un composé qui ne pénètre pas dans les cellules comme le sucrose, on peut obtenir une déshydratation partielle au dessus de $0^{\circ} \mathrm{C}$ et utiliser des vitesses de congélation plus grandes (méthode $A_{3}$ ). Enfin, en combinant l'action de plusieurs cryoprotecteurs on peut, avec des grandes vitesses de congélation (méthode A4), empêcher la formation de tout cristal de glace aussi bien à l'intérieur qu'à l'extérieur des cellules (vitrification). 
geant les structures cellulaires. Pour congeler les cellules, il faut donc contrôler les mouvements de l'eau pour limiter à la fois l'élévation de concentration en électrolytes et l'importance de la phase cristalline. En jouant sur les vitesses de congélation et de décongélation, on peut contrôler (au moins en partie) la formation de la glace intracellulaire et la croissance des cristaux. Mais ce n'est qu'en modifiant les propriétés physicochimiques des solutions par l'addition de cryoprotecteurs que l'on peut maintenir la viabilité de la cellule. Ces cryoprotecteurs, tel le glycérol, dont l'emploi avec des spermatozoïdes permit de congeler pour la première fois avec succès des cellules de mammifères [5], sont des solvants organiques qui ont une forte affinité de liaison avec l'eau : ils réduisent la vitesse de croissance des cristaux et en modifient la forme [6]. Ils agissent aussi directement sur la cellule et leur mode d'action (encore mal compris) implique notamment des associations avec les membranes et des modifications de la composition du milieu cellulaire [7]. Aujourd'hui, les propriétés de ces cryoprotecteurs sont utilisées de façon empirique : les données (physico-chimiques et biochimiques) actuellement disponibles sur l'interaction à différentes températures de ces substances avec les composés du milieu cellulaire, comme les protéines [8], n'ont pas encore été systématiquement mises à profit pour congeler un système cel- lulaire donné.

\section{Méthodes}

\section{de congélation}

Les premiers succès obtenus avec des embryons de souris ont montré que, contrairement à d'autres types cellulaires, la vitesse de congélation optimum pour la survie est très faible et de l'ordre de 0,3 à $I^{\circ} \mathrm{C} / \mathrm{min}[\mathrm{I}]$. La perméabilité à l'eau des cellules embryonnaires diminue en effet rapidement, et un refroidissement lent permet une déshydratation suffisante pour empêcher la formation d'une quantité de glace intracellulaire léthale.

Dans ces conditions, la survie n'est possible que si les embryons sont réchauffés également progressive- ment (4 à $\left.25^{\circ} \mathrm{C} / \mathrm{min}\right)$, ceci pour permettre la réhydratation de la cellule au cours de l'élévation de la température. La première méthode de congélation proposée pour congeler les embryons était une méthode dite "lente" demandant plusieurs heures ( 3 à $4 \mathrm{~h}$ ) pour amener progressivement, à l'aide d'un appareillage approprié, les embryons à la température de l'azote liquide (figure I, AI).

A partir des travaux effectués avec l'embryon de brebris [9], puis de souris [10], on s'aperçut que l'on pouvait raccourcir de moitié la durée de la congélation en plongeant les embryons dans l'azote liquide après refroidissement lent jusqu'à seulement $-40^{\circ} \mathrm{C} \quad(f i$ gure $\left.I, \mathrm{~A}_{2}\right)$. Des cristaux de glace intracellulaire se forment alors quand on passe de -40 à $-196^{\circ} \mathrm{C}$. Mais ces cristaux très petits seront sans effet immédiat sur les cellules si celles-ci sont décongelées très rapidement (plusieurs centaines de degrés par minute), afin d'éviter toute recristallisation. Cette deuxième méthode est parfois qualifiée de "rapide": en pratique, les vitesses de décongélation élevées sont obtenues en plaçant simplement le tube contenant les embryons directement de l'azote liquide dans un bain marie à 25 ou $37^{\circ} \mathrm{C}$.

Mais l'embryon peut être aussi partiellement déshydraté à température ambiante sans que sa viabilité ultérieure en soit affectée; cette déshydratation partielle est obtenue simplement par exposition à une solution de sucrose. Ce composé en effet ne pénètre pas dans les cellules et la membrane plasmique réagit comme un osmomètre, la sortie de l'eau contribuant à augmenter la concentration en solutés à l'intérieur des cellules, pour se rapprocher de l'équilibre osmotique. Dans ces conditions, le volume des cellules peut être réduit de plus de la moitié de sa valeur initiale, sans compromettre la reprise du développement après le retour à l'état normal [12]. Les mouvements de l'eau dans et hors de la cellule étant beaucoup plus rapides que ceux des solutés, si on place les embryons d'abord dans une solution contenant le cryoprotecteur perméable (glycérol ou propanédiol) puis temporai- rement dans une solution contenant du sucrose, on peut, tout en réalisant une déshydratation partielle des cellules à température ambiante augmenter rapidement leur concentration en cryoprotecteur. On se trouve placé dans des conditions qui favorisent la congélation du milieu cellulaire en l'absence de phase cristalline, puisque la vitesse de formation et de croissance des cristaux diminue rapidement quand la concentration du cryoprotecteur augmente. On peut alors obtenir la vitrification du milieu cellulaire, ce qui définit une $3^{\mathrm{e}}$ méthode de congélation des embryons (figure $I, \mathrm{~A}_{3}$ ).

Enfin, on peut chercher à empêcher la formation de tout cristal de glace non seulement à l'intérieur, mais aussi à l'extérieur des cellules. Cette vitrification totale des échantillons est obtenue en utilisant non plus un seul mais un mélange de cryoprotecteurs dont on combine les propriétés [13]. Ces mélanges sont aujourd'hui définis de façon empirique et l'on s'efforce de réduire leur effet toxique en diminuant la durée d'exposition aux cellules avant congélation ( figure $I, \mathrm{~A}_{4}$ ).

Quelque soit la méthode de congélation employée, les conditions de retrait du cryoprotecteur après décongélation (figure 2) jouent un rôle important dans la survie des cellules. Les effets exercés par le froid sur les membranes cellulaires modifient leur perméabilité, donc leurs propriétés osmotiques. Ces modifications, si elles ne sont pas trop importantes, peuvent être réversibles et les membranes retrouvent leurs propriétés initiales, notamment après une courte période de culture in vitro.

\section{Apport de l'expérience animale}

Les données actuellement publiées sur la congélation de l'embryon humain sont indiquées dans le tableau I. Ces données portent encore sur un nombre limité d'essais. Toutefois, à partir des travaux menés activement par quelques équipes dans le monde, on peut dégager plusieurs caractéristiques de l'embryon humain et les comparer à celles mieux connues des embryons des autres espèces. 


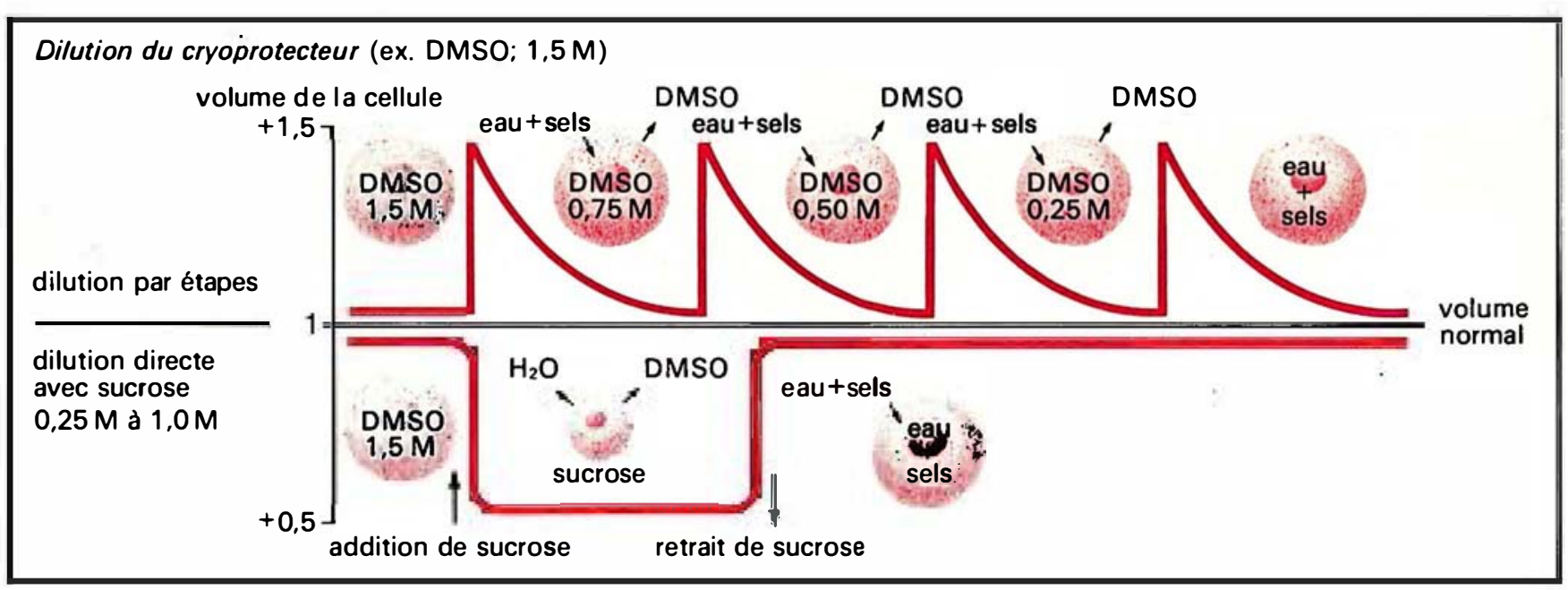

Figure 2. Méthodes utilisées pour la dilution du cryoprotecteur. Le retrait du cryoprotecteur après décongélation est nécessaire pour assurer la survie des embryons. Ce retrait est effectué le plus souvent à température ambiante, soit par étapes en plafant les embryons dans un gradient décroissant du cryoprotecteur (exemple: le DMSO), soit directement en exposant les embryons à une solution de sucrose qui, ne pénètrant pas dans les cellules, permet par un effet osmotique la sortie progressive du cryoprotecteur. C'est souvent au cours de cette opération finale de la congélation que se produisent des dégâts cellulaires importants.

\begin{tabular}{|c|c|c|c|c|c|c|c|c|c|}
\hline \multirow{5}{*}{ réf. } & \multicolumn{9}{|c|}{ SURVIE DE L'EMBRYON HUMAIN APRĖS CONGÉLATION DANS L'AZOTE LIQUIDE } \\
\hline & \multicolumn{2}{|c|}{$\begin{array}{l}\text { caractéristiques } \\
\text { de la congélation }\end{array}$} & \multicolumn{3}{|c|}{$\begin{array}{c}\text { survie in vitro } \\
\text { nombre }(\%) \text { d'embryons }\end{array}$} & \multicolumn{4}{|c|}{$\begin{array}{l}\text { survie in vitro } \\
\text { nombre : }\end{array}$} \\
\hline & \multirow{3}{*}{$\begin{array}{c}\text { stade } \\
\text { de } \\
\text { l'embryon }\end{array}$} & \multirow{3}{*}{$\begin{array}{l}\text { cryo- } \\
\text { protecteur } \\
\text { et méthode } \\
(a)\end{array}$} & \multirow[t]{3}{*}{ décongelés } & \multirow{2}{*}{\multicolumn{2}{|c|}{$\begin{array}{c}\text { dont } \\
\text { la proportion } \\
\text { de cellules } \\
\text { intactes est }\end{array}$}} & \multirow{3}{*}{$\begin{array}{l}\text { d'embryons } \\
\text { transplantés }\end{array}$} & \multirow{3}{*}{$\begin{array}{c}\text { de } \\
\text { patients }\end{array}$} & \multicolumn{2}{|c|}{ de grossesses } \\
\hline & & & & & & & & \multirow[b]{2}{*}{$\begin{array}{l}\text { initiées } \\
(c)\end{array}$} & \multirow[b]{2}{*}{ confirmées } \\
\hline & & & & $>50 \%$ & $100 \%$ & & & & \\
\hline [14] & $4-8 \mathrm{cel}$ & $\begin{array}{l}1 \text {-2 propa- } \\
\text { nédiol } \\
\text { rapide }\end{array}$ & 36 & $21(58)$ & $9(25)$ & - & - & - & - \\
\hline [15] & 4-8 cel & $\begin{array}{l}\text { DMSO } \\
\text { lente }\end{array}$ & 136 & $68(50)$ & $26(25)$ & 68 & 45 & 9 & 7 \\
\hline [16] & 4-16 cel & $\begin{array}{l}\text { glycérol } \\
\text { rapide }\end{array}$ & 15 & $8(53)$ & $2(25)$ & 15 & 11 & 2 & 2 \\
\hline [17] & $\begin{array}{l}\text { blasto- } \\
\text { cystes }\end{array}$ & $\begin{array}{l}\text { glycérol } \\
\text { rapide }\end{array}$ & 30 & 11 (36. & 6) $(b)$ & 23 & 11 & 2 & 1 \\
\hline
\end{tabular}

(a) refroidissement à $0,3^{\circ} \mathrm{C} / \mathrm{min}$ jusqu'à $-60^{\circ} \mathrm{C}$ dans le cas de la méthode "lente "et jusqu'à -30 à $-40^{\circ} \mathrm{C}$ dans le cas de la méthode "rapide "; (b) survie estimée par la réexpansion du blastocyste en culture pendant 2 à 25 heures après la décongélation; (c) grossesses décelées par dosage d'HCG (grossesses biochimiques). 
Comme pour la souris, la lapine et la vache, l'embryon humain peut être congelé avec succès à tous les stades de son développement, depuis le stade une cellule jusqu'au stade blastocyste. Les congélations sont effectuées surtout aux stades 4 et 8 cellules, correspondant aux stades où sont réalisées les remises en place après fécondation in vitro. Mais la survie semble possible au stade 2 pronucléi, soit vingt heures après l'insémination [14], ou au stade blastocyste avancé, c'est-à-dire 7 jours après fécondation in vitro [17]. Toutefois, comme pour d'autres espèces telles la souris [I8], la durée de la culture in vitro avant congélation tend à réduire le taux de survie des embryons après décongélation ( figure 3). De ce point de vue, l'embryon humain [I4] apparaît donc devoir être congelé aux stades très précoces de son développement.

L'embryon humain survit après exposition à différents cryoprotecteurs comme le DMSO, le glycérol ou le propanédiol. La perméabilité au glycérol est très faible aux stades 2 cellules et 8 cellules : pour assurer la pénétration de ce cryoprotecteur, il faut des temps d'exposition longs et lors de la dilution après décongélation, un gonflement marqué des blastomères dû̀ ù une réhydratation rapide, est fréquemment observé [ I 5]. Au stade blastocyste par contre, la perméabilité au glycérol est élevée [I7] et on retrouve là une propriété commune aux blastocystes des mammiferes. Le DMSO paraît mieux convenir que le glycérol, non seulement parce qu'il pénètre plus facilement les cellules, mais aussi parce que, largement utilisé jusqu'à présent pour la congélation des embryons de souris, on sait qu'il n'induit aucune perturbation génétique sur l'embryon [19]. Le propanédiol est également un bon cryoprotecteur : ce composé pénètre facilement les blastomères de l'embryon humain, présente l'avantage de limiter la formation des cristaux de glace à la congélation et rend plus stable l'état vitreux obtenu à basse température. A concentration élevée, ce composé, encore peu utilisé, est apparemment moins toxique pour les cellules que le glycérol ou le DMSO [20]. Les possibilités de survie de l'embryon humain après décongélation dépendent étroitement de son état de développement au moment de la congélation. Les blastomères dont le noyau est en interphase (visible à l'observation au microscope optique) semblent mieux résister aux effets de la conservation que les blastomères dont le noyau est en cours de division mitotique [14]. L'absence d'anomalies morphologiques au moment de la congélation augmente les chances de succès : comme pour l'embryon bovin ( $f i$ gure 4), le tri des embryons à congeler permet d'augmenter le taux de survie après conservation [2I].

Dans l'état actuel des recherches, la technique de congélation des embryons surnuméraires off re encore peu de chances supplémentaires de

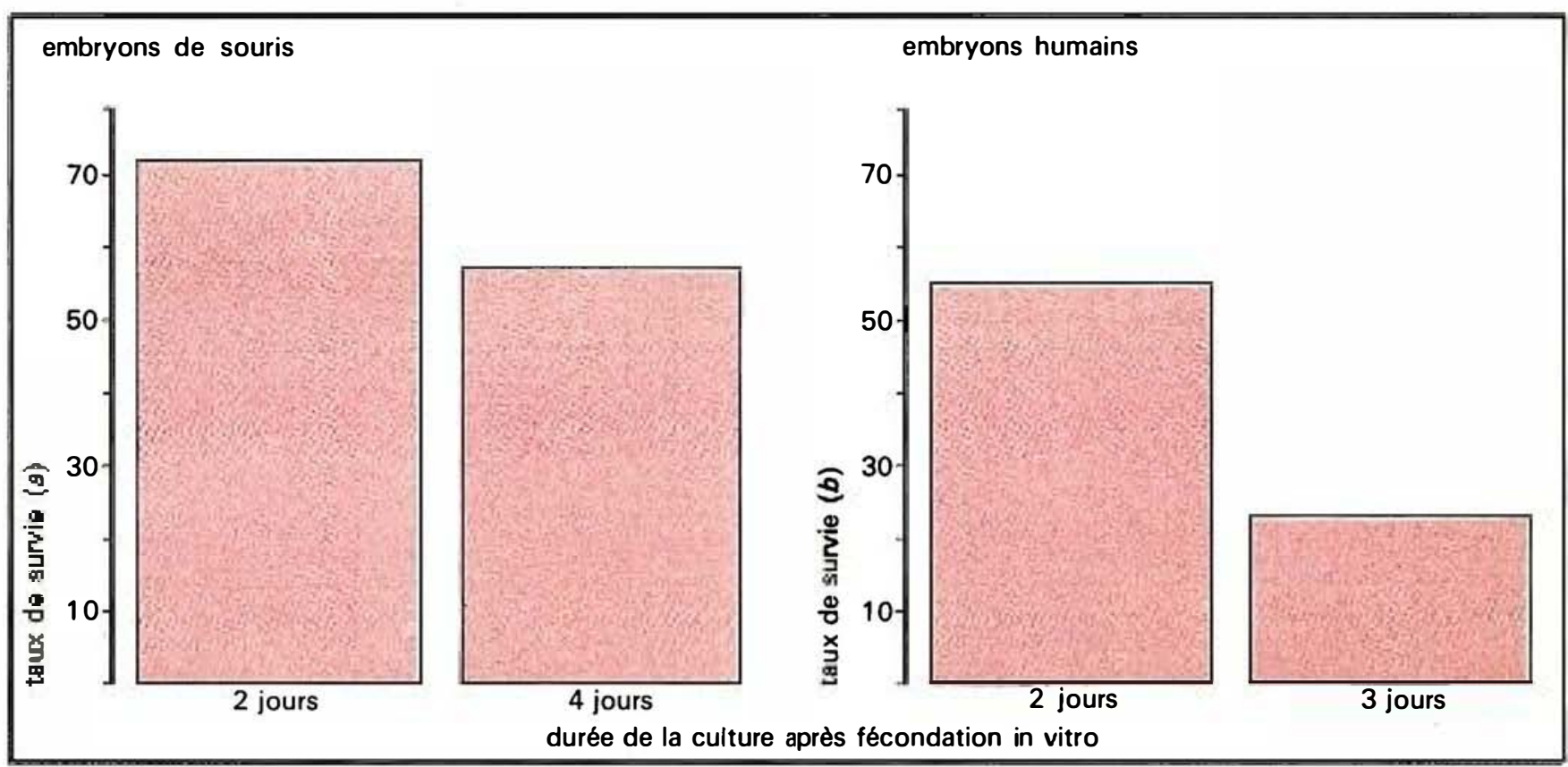

Figure 3. Effet de la durée de culture avant congélation sur le taux de survie d'embryons humains fécondés in vitro [14]. Comparaison avec l'animal (souris) [18]. Chez l'homme comme chez la souris le pourcentage d'embryons fécondés in vitro qui se développent en jeunes ou en fatus viables est réduit environ aux trois quarts de sa valeur initiale quand on double la durée de la culture avant congélation : il apparaît donc préférable de congeler l'embryon humain aux stades très précoces de son développement.

(a) survie estimée par le pourcentage relatif d'embryons congelés qui se développent en jeunes ou faetus viables après transfert (témoins = I00); (b) survie estimée par le pourcentage relatif d'embryons congelés dont plus de la moitié des cellules est intacte après décongélation (témoins $=100$ ). 


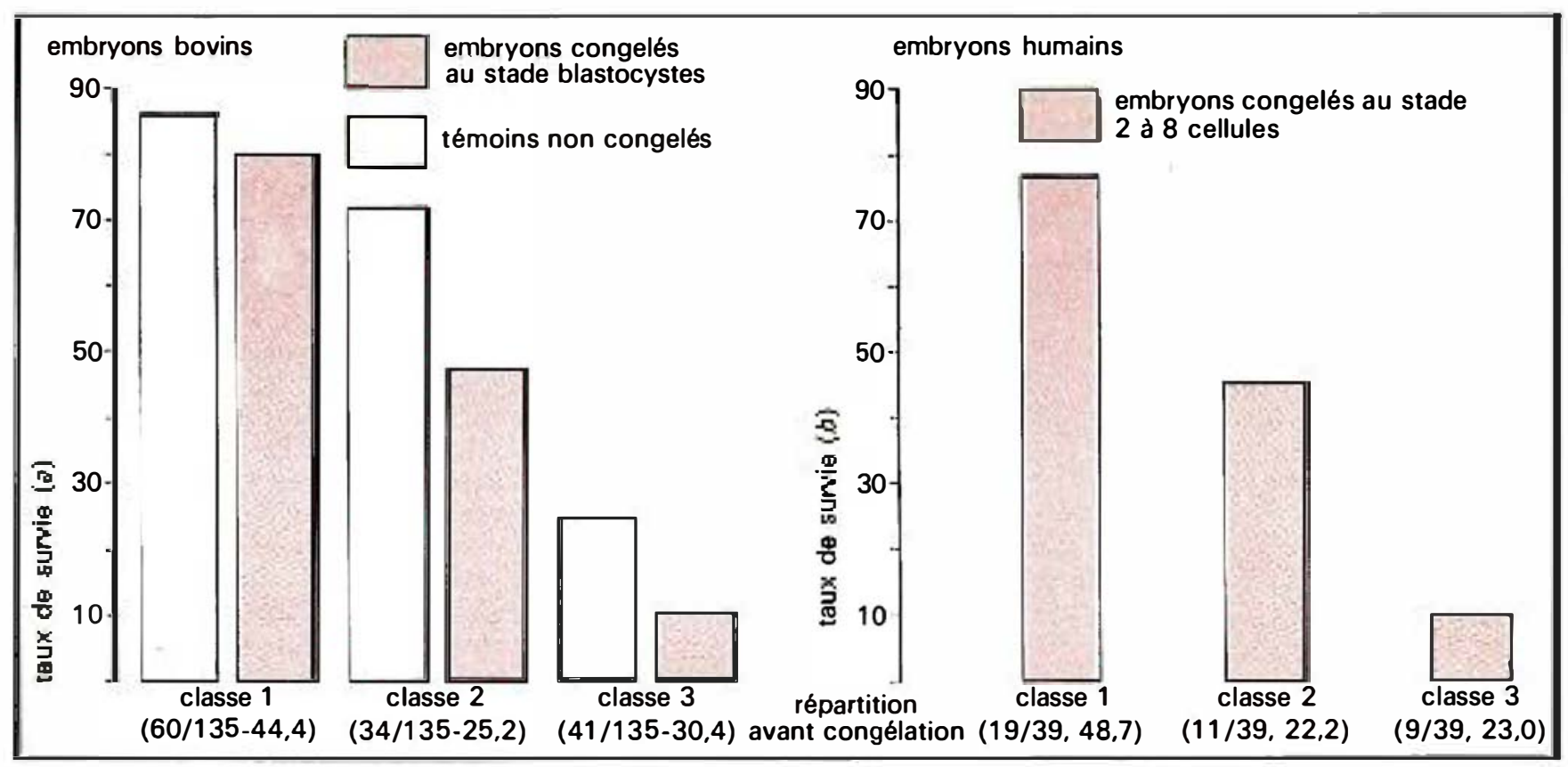

Figure 4. Effet sur le taux de survie après décongélation d'une sélection avant congélation des embryons humains [14]. Comparaison avec l'animal (vache) [2I]. Le tri des embryons avant congélation est actuellement effectué à partir de critères morphologiques : la classe I correspond aux embryons qui n'ont aucune anomalie visible; la classe 2 aux embryons dont une ou quelques cellules sont dégénérées et la classe 3 aux embryons en retard dans leur développement. Cette sélection laisse une grande place à la subjectivité de l'opérateur mais permet de regrouper les meilleurs embryons qui donneront un taux de survie supérieur à $70 \%$. Le pourcentage d'embryons de classe I varie considérablement d'un individu à l'autre; chez la vache, certaines femelles peuvent régulièrement produire des embryons qui resteront tous intacts après décongélation.

(a) survie estimée par le pourcentage d'embryons qui se développent normalement en culture pendant 72 heures à $37^{\circ} \mathrm{C}$; (b) survie estimée par le pourcentage d'embryons dont plus de la moitié des cellules sont intactes après décongélation.

grossesse aux patientes : les données publiées montrent que dix grossesses seulement $(5,5 \%)$ ont pu être établies à partir des i 8 I embryons décongelés en vue de leurs transferts (tableau I) [15-16-I7]. Ce taux de réussite est deux fois plus faible que celui obtenu après remise en place d'embryons non congelés (en moyenne $12 \%$ de réussite par cycle de traitement avec remise en place de deux embryons). Des méthodes de conservation plus efficaces doivent être définies. Mais le fait que des grossesses et naissances normales aient pu être établies à partir d'embryons dont plusieurs blastomères avaient été endommagés par la congélation souligne l'extraordinaire aptitude à la survie de l'embryon humain (figure 5, voir p. 33). Comme chez l'animal, il apparaît que les dégâts dus à la conservation exercent un effet de " tout ou rien" sur le développement; ces dégâts ne contribuent pas $m / s n^{\circ}$ I vol. 2 janvier 86 à augmenter le taux d'ancmalies ou de malformations des jeunes nés après transfert d'embryons congelés.

Les données accumulées depuis plus de 12 ans chez l'animal indiquent que la durée du stockage à basse température n'affecte pas la viabilité ultérieure des embryons : chez la souris, après transfert d'embryons stockés à $-196^{\circ} \mathrm{C}$ quelques heures seulement ou pendant plus de 6 ans, des taux de naissances semblables ont été obtenus. Les principales réactions qui peuvent se produire à ces températures pourraient être induites par l'accumulation, au cours du temps, des effets des radiations ionisantes; or, on a montré que l'exposition des embryons congelés pendant plusieurs mois à l'équivalent de 2000 années de radiations, n'affecte pas le taux de survie, ni le taux de malformations à la naissance [19]. Ces données rendent très peu probable l'existence d'un effet de la conservation de l'embryon dans l'azote liquide sur l'aptitude au développement tant physique que psychique de l'enfant. Enfin, on peut souligner que la simplification des méthodes de congélation que l'on constate actuellement chez l'animal permet une banalisation de la technique qui, il y a quelques années, était considérée uniquement comme un outil de laboratoire. L'embryon congelé fait maintenant partie des moyens qu'utilise l'industrie de l'élevage, notamment bovin, pour assurer la diffusion des gènes de ses meilleures femelles. En I984, les quelques 200 à 300 firmes spécialisées dans le transfert d'embryons dans le monde ont effectué plus de 400000 interventions, dont le quart environ avec des embryons congelés : ceux-ci sont conservés maintenant, de plus en plus fréquemment, dans des petites "paillettes " d'un quart de millilitre (dérivées de celles 
utilisées pour l'insémination artificielle). Un code d'identification très précis a été défini au niveau international afin de permettre le bon fonctionnement des transactions commerciales entre pays. Cette banalisation devrait contribuer au développement de ces techniques chez l'homme.

\section{Congélation de l'ovocyte}

On ne dispose encore d'aucune donnée publiée sur les possibilités de développement de l'ovocyte humain après décongélation. La souris est la seule espèce où l'on a pu obtenir quelques jeunes vivants à partir d'ovocytes congelés dans l'azote liquide puis fécondés in vitro après décongélation [22]; les taux de survie sont très faibles $(7 \%)$ par rapport à ceux obtenus avec des embryons au stade 8 cellules congelés avec la même méthode (plus de $40 \%$ ). Contrairement à l'embryon, une des principales interrogations sur la congélation de l'ovocyte concerne le risque d'induction d'anomalies chromosomiques. Au moment de l'ovulation en effet, l'ovocyte est au stade métaphase 2 de la méiose : la membrane nucléaire n'est pas formée, et les chromosomes sont regroupés sur les fibres de fuseau de division formées de plusieurs types de microtubules associés à des protéines. Des études (en ultrastructure) de l'ovocyte de souris ont montré que le fuseau de microtubules se dépolymérise en dessous de $4^{\circ} \mathrm{C}$ et que, après réchauffement, des anomalies dans la position des chromosomes peuvent se produire quand le fuseau se reconstitue [23]: ces anomalies peuvent être à l'origine de monosomies ou trisomies. L'ovocyte mûr apparaît donc particulièrement sensible aux effets de refroidissement. Des études récentes montrent toutefois que l'utilisation de cryoprotecteurs permet de prévenir les anomalies liées à la non disjonction des
Une autre voie de recherches encore non explorée (Testart, communication personnelle), consiste à congeler l'ovocyte avant qu'il ait atteint sa maturité, c'est-à-dire quand la membrane nucléaire (vésicule germinative) est encore présente et peut "protéger " les chromosomes. Mais dans ce cas, il faut pouvoir maîtriser toutes les étapes de la maturation in vitro.

Les modalités de disjonction des chromosomes en fin de méiose ne sont pas les mêmes chez l'homme et dans les espèces animales utilisées comme modèle d'étude : les expériences sur les ovocytes animaux ne peuvent donc que compléter les essais chez l'homme; une bonne connaissance des modifications cellulaires induites par la conservation à basse température est nécessaire et doit précéder toute tentative de remise en place d'un ovocyte humain congelé.

\section{Au cour du débat éthique}

$\mathrm{Si}$, comme c'est le cas le plus souvent aujourd'hui, on reconnait le bien-fondé de la procréation artificielle comme remède à la stérilité, on doit considérer que la congélation de l'embryon et de l'ovocyte humains constitue avant tout un moyen d'améliorer les traitements qui sont mis en œuvre dans le cadre de la FIVETE. En effet, congeler c'est introduire une dissociation supplémentaire dans la chaîne d'opérations qui, depuis la stimulation hormonale de l'ovaire jusqu'au transfert de l'embryon après fécondation in vitro, permettent l'établissement d'une grossesse. Cette dissociation dans le temps entre la production des gamètes et la fécondation (congélation de l'ovocyte) ou entre le début du développement embryonnaire et la grossesse (congélation de l'embryon) permet non seulement de mieux décider du moment où sera établi cette grossesse (équilibre hormonal, état de santé général...) mais aussi de réserver pour d'éventuelles tentatives ultérieures les embryons surnuméraires non remis en place au premier essai, épargnant ainsi à la

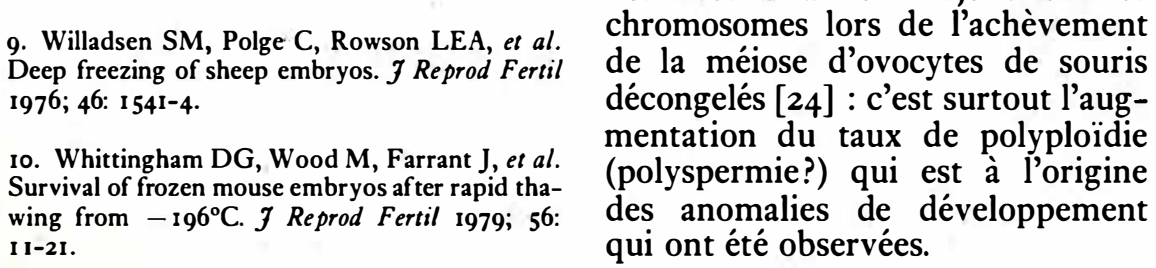

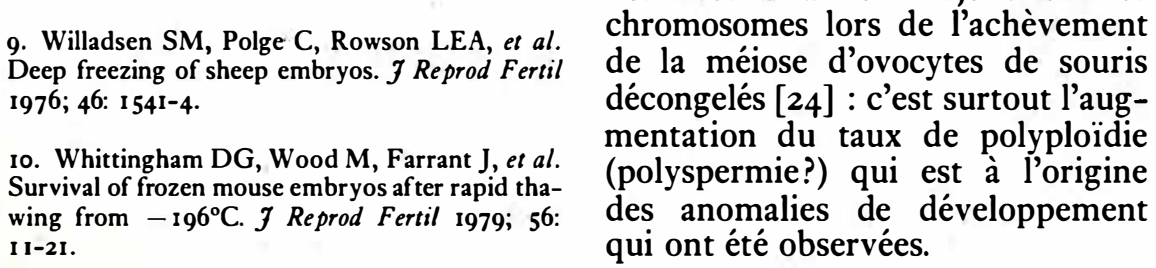

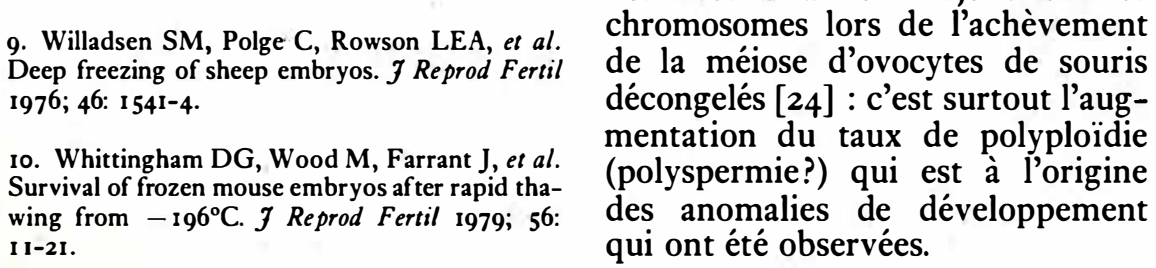

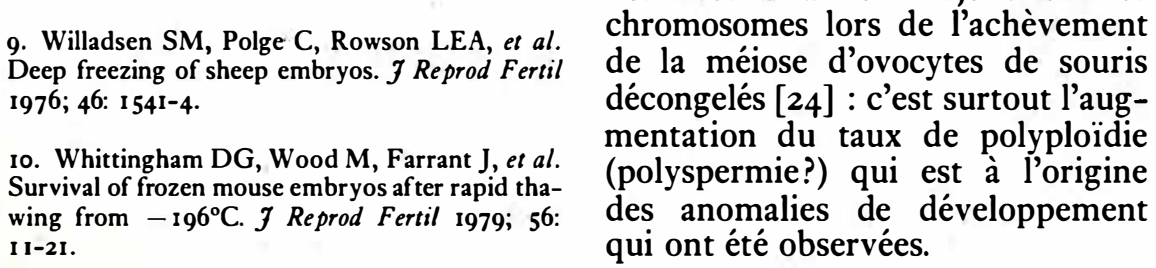

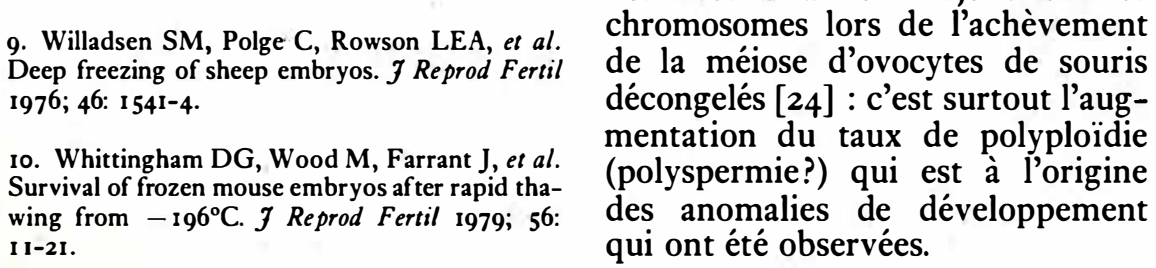

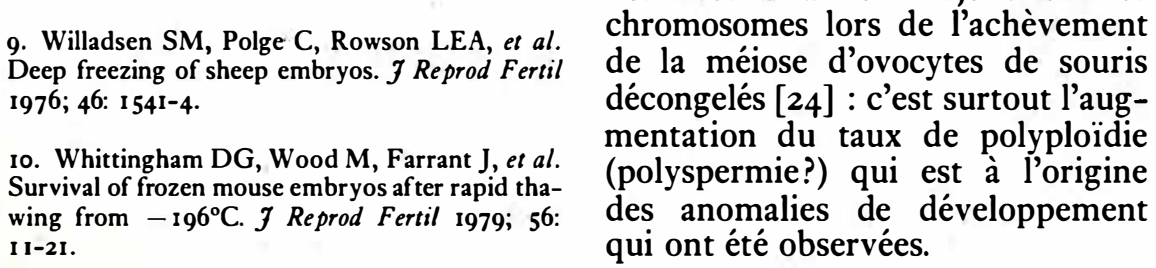

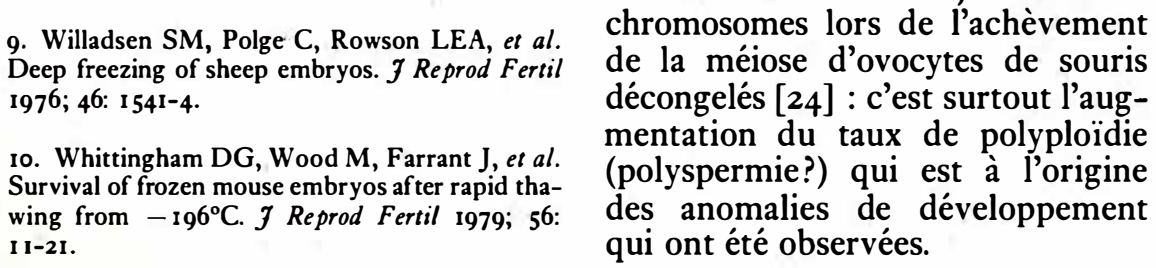

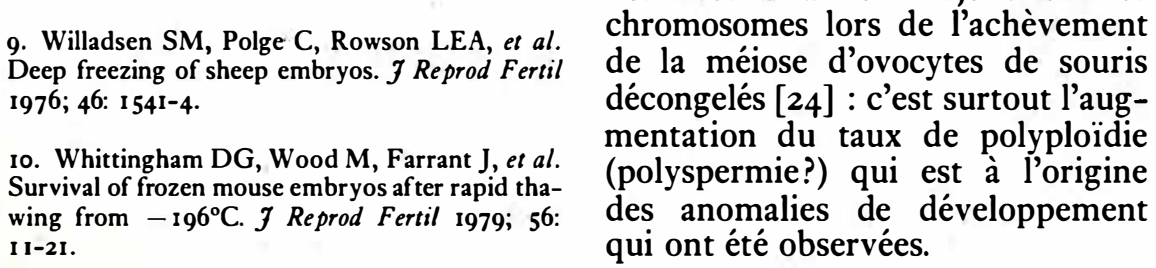

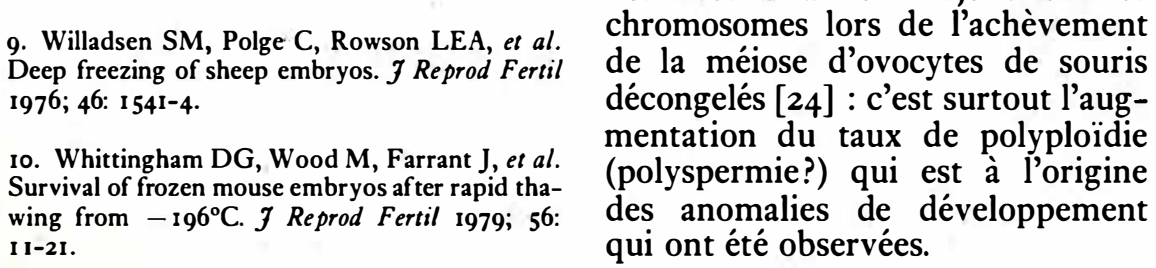

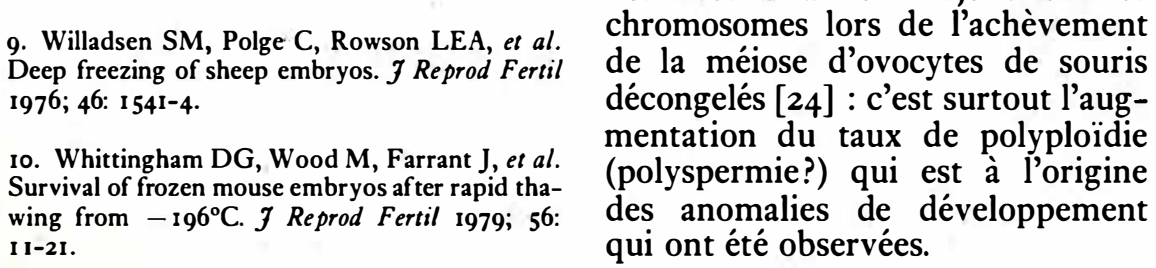

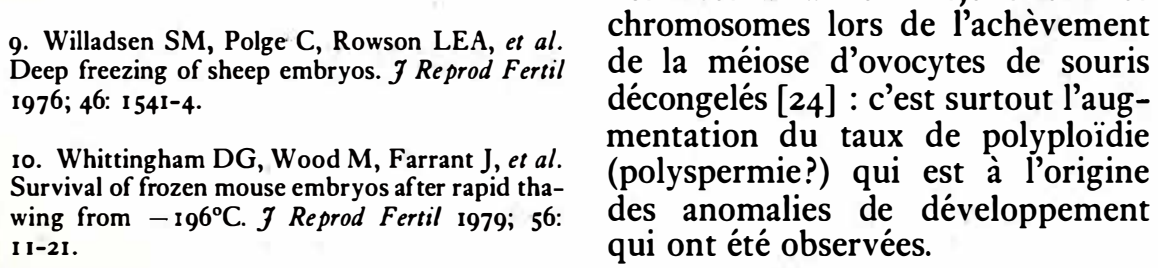


femme de subir plusieurs fois la partie du traitement destinée à la collecte des ovocytes (traitements hormonaux, coliscopie etc.).

Mais, congeler c'est aussi s'engager à décider à un moment donné du sort que l'on réserve à l'embryon en surplus. C'est donc prendre position sur le don d'ovocyte et le don d'embryon, problèmes moraux d'importance posés par la pratique de la FIVETE (don à un couple, à une femme, à la recherche...).

Congeler, c'est enfin constituer une banque dont la gestion et les conditions d'utilisation dépassent largement la responsabilité des seuls biologistes ou médecins. Ceux-ci se trouvent confrontés à la difficulté de définir des limites aux recherches. A partir d'un stock d'embryons ou d'ovocytes humains disponibles, peut-on moralement envisager d'appliquer des techniques pratiquées déjà sur les espèces animales : production de jumeaux par division de l'embryon à un stade très précoce de son développement; biopsie permettant de déterminer à partir de quelques cellules si l'embryon est exempt d'anomalies génétiques; substitution de noyau créant un embryon à deux mères génétiques (noyau et cytoplasme); transgénose qui, par injection de séquences spécifiques d'ADN dans le noyau, modifie le patrimoine génétique de l'individu; ou encore établissement de lignées cellulaires...?

Aujourd'hui commence peu à peu à être défini un ensemble de principes qui tente de donner à nos sociétés les moyens de surmonter les enjeux nés de la progression de nos connaissances dans le domaine de la reproduction humaine. Le rapport de la Commission Warnock [25] créée en Angleterre en juillet I982 constitue à ce jour le document le plus complet établi sur cette question. Dans plusieurs pays, dont la France, on propose que les embryons congelés ne soient pas $\mathrm{Ia}^{-}$ propriété de leurs parents qui garderaient le droit de disposer de leur destinée mais ne pourraient pas les vendre (...). On reconnait à l'organisme chargé de la conservation un droit sur l'utilisation de ces embryons en cas de décès de l'un ou des deux parents. On envisage un $m / s n^{\circ}$ I vol. 2 janvier 86
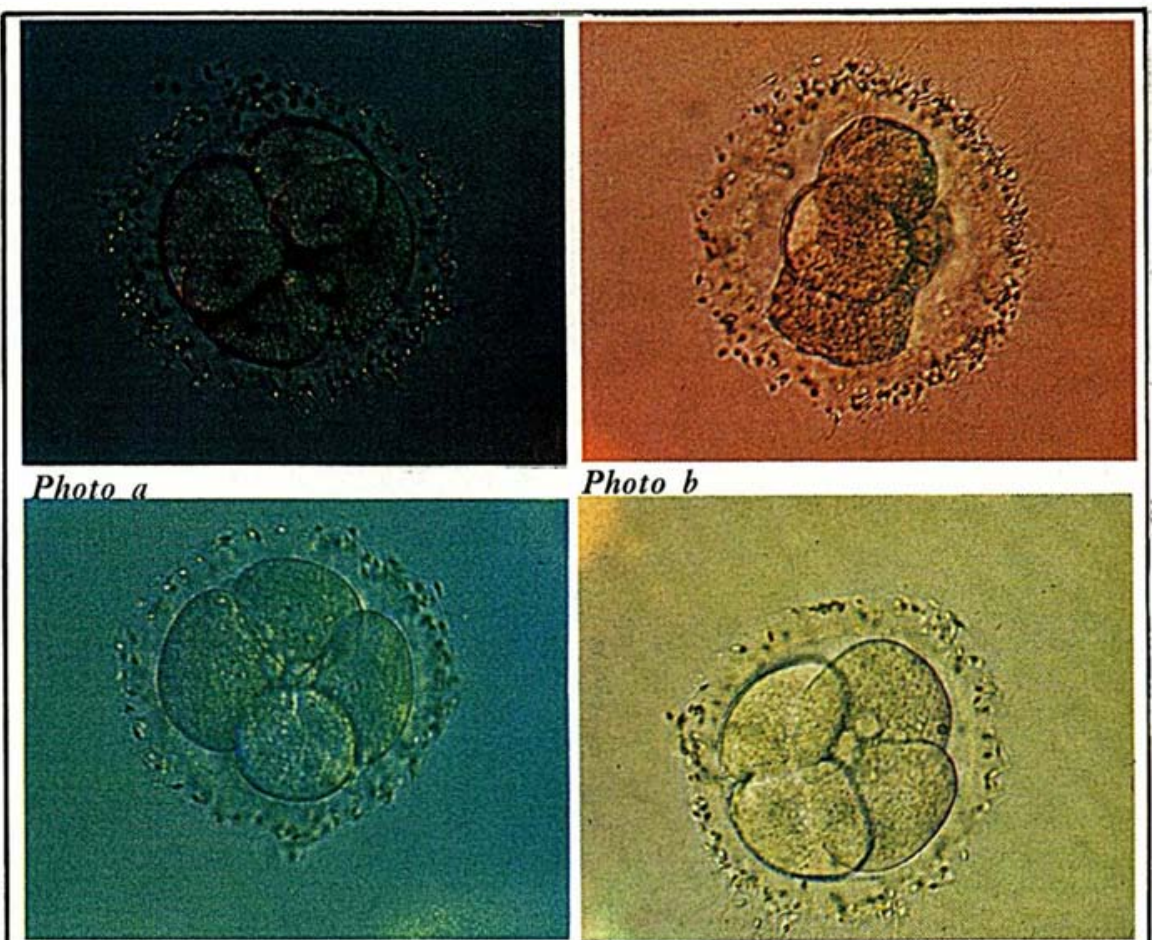

Photo c

Photo d
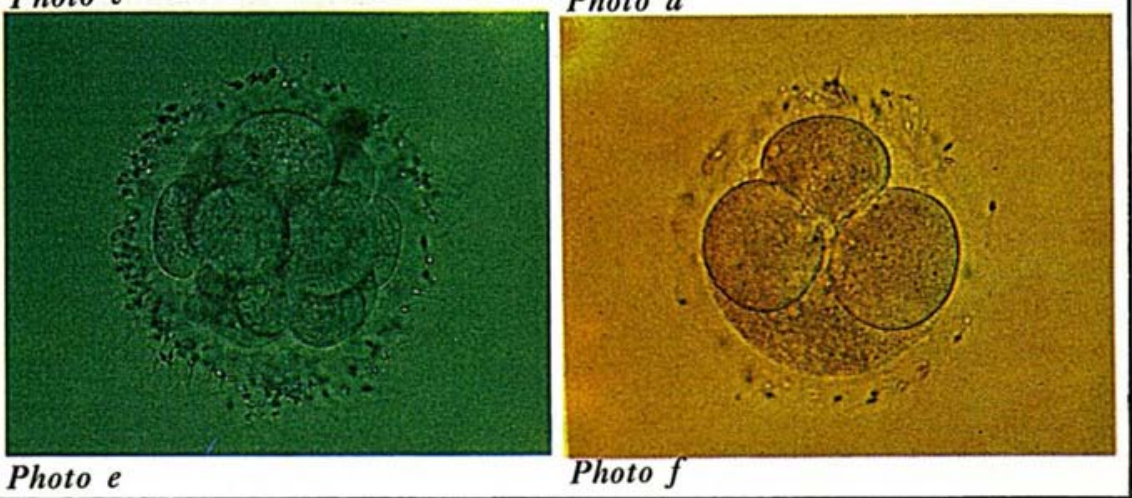

Photo e

Photo f

Figure 5. Embryons humains avant et après congélation.

Photo a : Embryon humain au stade 4 cellules. Cet embryon qui ne présente aucune anomalie mor phologique visible est un bon candidat pour la congélation. Sur la zone pellucide qui l'entoure, adhérent encore des cellules du follicule (corona radiata) et des spermatozoïdes. Cliché B. Lassalle, Inserm UI87.

Photo b et $c$ : Le même embryon au cours du traitement qui précède l'abaissement de la température. L'embryon est exposé à une solution contenant 10 à $15 \%$ de propanédiol, un cryoprotecteur efficace qui favorise la formation de glace amorphe (sans cristaux). Ce cryoprotecteur se substitue à une partie de l'eau cellulaire dont il modifie les propriétés physico-chimiques. Il pénètre dans les cellules plus rapidement que l'eau n'en sort, ce qui provoque d'abord leur retraction (photo b) puis leur retour à l'état antérieur (photo c). Clichés B. Lassalle, Inserm U I87.

Photos $d$ et $e$ : Le même embryon après décongélation. Le cryoprotecteur a été retiré à l'aide d'une solution de sucrose et tous les blastomères sont intacts. (photo $d)$. Après $48 \mathrm{~h}$ de culture in vitro, cet embryon s'est développé normalement et a 10 cellules (photo e). Clichés B. Lassalle, Inserm U I87.

Photo $f$ : Exemple de dégâts cellulaires sur un embryon humain après décongélation au stade de 4 cellules. Les dégâts visibles concernent ici un seul blastomère. La congélation exerce un effet de "tout ou rien" sur l'aptitude au développement des cellules et l'expérience animale montre que des dégâts localisés ne contribuent pas à augmenter le taux d'anomalies ou de malformation des jeunes nés après transfert. Cliché B. Lassalle, Inserm U 187. 


\section{REFERENCES}

11. Rall WF, Reid DS, Polge C. Analysis of slow warming injury of mouse embryos by cryomicroscopal and physico-chemical methods. Cryobiology 1984; 21 : 106-21.

12. Renard JP, Bui-Xuan-Nguyen N, Garnier V Two-step freezing of two-cell rabbit embryos after partial dehydration at room temperature. $\mathcal{J}$ Reprod Fertil 1984; 71 : 573-80.

13. Rall WF, Fahy GM. Icc free cryopreservation of mouse embryos at $-196^{\circ} \mathrm{C}$ by vitrification. Nature 1985; 313: 573-5.

14. Lassalle B, Testart J, Renard JP. Human embryos features influencing the success of cryopreservation using I-2 propanediol. Fertil Steril 1985 (in press).

15. Mohr LR, Trounson A, Freemann L. Deep freezing and transfer of human embryos. 7 In Vitro Fertil Steril 1984; 42: 293-6.

16. Zeilmaker GH, Alberda ATH, Van Gent I, et al. Two pregnancies following transfer of intact frozen-thawed cmbryos. Fertil Steril 1984; 42: 293-6.

17. Cohen J, Simons RF, Edwards RG, et al. Pregnancies following the frozen storage of expanding human blastocysts. I In Vitro Fertil Embr yo Trans 1985; 2: 59-64.

18. Massip A, Van Der Zwalmen P, Puissant F, et al. Effects of in vitro fertilization, culture, freezing and transfer on the ability of mouse embryos to implant and survive. 7 Reprod Fertil 1984; 71: 199-204

19. Glenister PH, Whittingham DG, Lyon MF. Further studies on the effect of radiation during the storage of frozen 8 cell mouse embryos at - 196 ${ }^{\circ}$ C. 7 Reprod Fertil 1984; 70: 229-34.

20. Renard JP. The cryopreservation of mammalian embryos. In: Testart J, Frydmann R, eds. Human in vitro fertilization: actual problems and prospects. Amsterdam: Elsevier, 1985: 201-8.

21. Renard JP, Heyman Y, Leymonie $P$, et al. Sucrose dilution: a technique for field transfer of in straw frozen bovine embryos. Theriogenology 1983; $19: 145$

22. Wittingham DG. Fertilization in vitro and the developpement to term of unfertilized mouse ovocytes previously stored at $-196^{\circ} \mathrm{C}$. 7 Reprod Fertil 1977; 49: 89-94.

23. Magistrini M, Szollozi D. Effect of cold and of isopropyl-N Phenylcarbamate on the second meiotic spindle of mouse ovocytes. Europ 7 Cell Biol 1980; 22: 699-707.

24. Wood M, Glenister PH, Wittingham DG. In $4^{\text {th }}$ world conference on in vitro Fertilization. Melbourne, 1985 (to be published).

25. Fecondation et embryologie humaines: rapport de la Commission d'enquête présidée par dame Mary Warnock concernant la fécondation et l'embryologie humaines. In : Paris : la documen- contrôle de ces organismes (tous les cinq ans en Angleterre) et l'on propose de fixer une durée maximale de conservation des embryons en vue terre, trois ans en Australie, et un an en France.

Rappelons que la congélation d'embryons a déjà provoqué un imbroglio éthique, juridique et scientifique en Australie, où le sort de deux embryons congelés présentés au public comme étant non seulement "orphelins" à la suite du décès accidentel de leurs parents génétiques, mais aussi riches " héritiers ", a provoqué un affrontement entre le Comité sur la fécondation in vitro du Ministère de la justice de Melbourne recommandant de détruire ces embryons, et une association défendant le droit à la vie, qui s'opposait à la réalisation de ce "meurtre".

\section{Conclusion}

La congélation de l'embryon humain apparaît aujourd'hui comme un des moyens cliniques dont doivent se doter les programmes de FIVETE définis pour répondre à la demande d'enfant de la part de couples stériles. L'efficacité des techniques est encore limitée, mais le développement récent des méthodes de congélation de l'embryon chez l'animal devrait rapidement apporter des améliorations chez l'homme. L'extension de cette utilisation d'embryons humains congelés soulève d'importants problèmes éthiques liés principalement à la durée à fixer pour le stockage, durée qui n'affecte apparemment pas les possibilités ultérieures de développement de l'embryon. La congélation de l'ovocyte humain, si elle s'avère possible, modifiera les conditions d'utilisation de cette technique qui deviendraient alors similaires à celles de l'insémination artificielle avec du sperme congelé bien définies actuellement. De nouvelles voies d'étude, privilégiant les caractéristiques physico-chimiques du milieu cellulaire et leurs modifications sous l'effet du froid, s'offrent aux chercheurs placés au cœur de cet enjeu fascinant mais redoutable que constitue pour nos sociétés la perspective d'une maîtrise de la reproduction humaine de leur transfert : dix ans en Angle-

\section{Summary}

Freezing of the human embryo is now being used to offer an additional possibility of pregnancy in patients treated by in vitro fertilization for infertility. The success rate is still low, about half as high as that of the tranfer of unfrozen "embryos. Several methods of freezing are available: they have been designed from knowledge acquired in recent years on the behaviour of embryonic cells at low temperature. Several hundreds of frozen human embryos have already been implanted throughout the world, and banks are being constituted for storage purposes. Development of research in this area raises important ethical questions, including the conditions of utilization of these banks and the duration of storage of the embryos.

\section{TIRES A PART}

Jean-Paul Renard : Unité de génétique des mammiferes, Institut Pasteur, 25, rue du Docteur-Roux, 75724 Paris cedex 15. 